\title{
AN EVEN BETTER REPRESENTATION FOR FREE LATTICE-ORDERED GROUPS
}

\author{
BY
}

\author{
STEPHEN H. MCCLEARY
}

\begin{abstract}
The free lattice-ordered group $F_{\eta}$ (of rank $\eta$ ) has been studied in two ways: via the Conrad representation on the various right orderings of the free group $G_{\eta}$ (sharpened by Kopytov's observation that some one right ordering must by itself give a faithful representation), and via the Glass-McCleary representation as a pathologically $o$-2-transitive $l$-permutation group. Each kind of representation yields some results which cannot be obtained from the other. Here we construct a representation giving the best of both worlds-a right ordering $\left(G_{\eta}, \leqslant\right)$ on which the action of $F_{\eta}$ is both faithful and pathologically $o$-2-transitive. This $\left(G_{\eta}, \leqslant\right)$ has no proper convex subgroups. The construction is explicit enough that variations of it can be utilized to get a great deal of information about the root system $\mathscr{P}_{\eta}$ of prime subgroups of $F_{\eta}$. All $\mathscr{P}_{\eta}$ 's with $1<\eta<\infty$ are $o$-isomorphic. This common root system $\mathscr{P}_{f}$ has only four kinds of branches (singleton, three-element, $\mathscr{P}_{f}$, and $\mathscr{P}_{\omega_{0}}$ ), each of which occurs $2^{\omega_{0}}$ times. Each finite or countable chain having a largest element occurs as the chain of covering pairs of some root of $\mathscr{P}_{f}$.
\end{abstract}

1. The Conrad representation has been used most extensively by Arora and McCleary [1], who studied centralizers of certain elements of $F_{\eta}$. The GlassMcCleary representation was exploited (and partially developed) by McCleary in [7], to which the present paper is a sequel. Familiarity with [7] (but not [1]) is assumed.

The Conrad representation [2] proceeds as follows: Given any right ordering $\left(G_{\eta}, \leqslant\right)$ of the free group $G_{\eta}$, the right regular representation $\varphi$ of $G_{\eta}$ preserves the order (but is not in general an $l$-permutation group). By the freeness of $F_{\eta}$ on the free generating set $\mathbf{x}$ (which generates $G_{\eta}$ as a group), $\varphi$ can be extended to a unique l-homomorphism into $A\left(\left(G_{\eta}, \leqslant\right)\right.$ ) (i.e., to a unique action on the chain $\left(G_{\eta}, \leqslant\right)$ ), namely

$$
w \bar{\varphi}=\left(\bigvee_{i} \bigwedge_{j} w_{i j}\right) \varphi=\bigvee_{i} \bigwedge_{j} w_{i j} \varphi .
$$

We shall refer to this as the natural action of $F_{\eta}$ on $\left(G_{\eta}, \leqslant\right)$. When this action is a representation (i.e., faithful), we shall call $\left(G_{\eta}, \leqslant\right)$ a representing right ordering.

Another kind of action of $F_{\eta}$ is usual transitive action on the chain $F_{\eta} / P$ of right cosets of a prime subgroup $P$ (namely $(P f) w=P(f w)$ ). When this action is a representation, $P$ is called a representing subgroup of $F_{\eta}$.

Received by the editors February 21, 1984.

1980 Mathematics Subject Classification. Primary 06F15.

Key words and phrases. Free lattice-ordered group, ordered permutation group, right ordered group. 
Kopytov's sharpening [4] of Conrad's representation begins with the assumption that $F_{\eta}$ has some transitive representation (known originally only for infinite $\eta$, but now known for all $\eta>1$ [7]). We shall make use of his proof that there must then exist a representing right ordering $\left(G_{\eta}, \leqslant\right)$, so we give (a somewhat streamlined version of) the proof here (Theorem 2 ), and then point out a very short route to the Conrad representation.

Inspection of the results which can be obtained only from the Conrad-Kopytov representation (and not from the Glass-McCleary representation) reveals that only one crucial feature of the representation is used: Every $e \neq w \in F_{\eta}$ maps to a nonidentity element in some transitive action on a chain $\Omega$ in which no (image of a) group word $e \neq g \in G_{\eta}$ fixes any point. (That $\Omega$ is a right ordering of $G_{\eta}$ does not matter.) The fact that this feature holds already for Conrad's representation explains why Kopytov's sharpening has not heretofore proved useful, and why we need a further sharpening to make it useful here.

This led to the search for a pathologically $o$-2-transitive representation $\left(F_{\eta}, \Omega\right)$ having this additional property. In fact, the property turns out to force $\Omega$ to be a right ordering $\left(G_{\eta}, \leqslant\right)$ of $G_{\eta}$. Thus success in this search amounts to proving the "best of both worlds" theorem (Main Theorem 3).

Finally we modify the proof of this result to describe the root systems $\mathscr{P}_{\eta}$ (Main Theorems $4 \mathrm{~A}-4 \mathrm{C}$.) Theorem $4 \mathrm{C}$, the version with $\eta>\omega_{0}$, along with many other results, requires that $\eta$ be regular and assumes the Generalized Continuum Hypothesis. (The cardinal number $\eta$ is regular if, as an initial ordinal number, it has no cofinal subset of cardinality less than $\eta$.)

Observe that whereas most previous results about free l-groups have been expected, even "obvious", this is far from true of the present results.

2. The best of both worlds. We begin by describing Kopytov's approach [4] which starts with a transitive representation $\left(F_{\eta}, \Omega\right)$ (see [7]) and constructs a representing right order $\left(G_{\eta}, \leqslant\right)$.

LEMMA 1 (Kopytov). In any transitive action $\left(F_{\eta}, \Omega\right)$, even $G_{\eta}$ acts transitively on $\Omega$.

Proof. Let $\alpha, \beta \in \Omega$. Then $\alpha w=\beta$ for some $w=\bigvee_{i} \wedge_{j} w_{i j} \in F_{\eta}\left(w_{i j} \in G_{\eta}\right)$. Now $\beta=\alpha\left(\bigvee_{i} \wedge_{j} w_{i j}\right)=\max _{i} \min _{j} \alpha w_{i j}=\alpha w_{i^{\prime} j^{\prime}}$ for some $w_{i^{\prime} j^{\prime}}$.

THEOREM 2 (KopyTOV). Let $\left(F_{\eta}, \Omega\right)$ be a transitive representation of $F=F_{\eta}$, and let $F_{\alpha}$ be the stabilizer subgroup of some $\alpha \in \Omega$. Pick any right ordering $\left(F_{\alpha} \cap G_{\eta}, \preccurlyeq\right)$ of the free group $F_{\alpha} \cap G_{\eta}$. Then a right ordering $\left(G_{\eta}, \leqslant\right)$ of $G_{\eta}$ is given by

$$
g_{1} \leqslant g_{2} \Leftrightarrow\left(\alpha g_{1}<\alpha g_{2} \text {, or } \alpha g_{1}=\alpha g_{2} \text { and } e \preccurlyeq g_{2} g_{1}^{-1}\right) \text {. }
$$

The natural action of $F_{\eta}$ on $\left(G_{\eta}, \leqslant\right)$ is faithful. Moreover, the sets $\Delta_{\beta}=\left\{g \in G_{\eta} \mid \alpha g=\right.$ $\beta\}(\beta \in \Omega)$ are the classes of a convex congruence $\mathscr{C}$ (with $\Delta_{\alpha}=F_{\alpha} \cap G_{\eta}$ ), and the representation $\left(F_{\eta}, \mathscr{C}\right)$ coincides with $\left(F_{\eta}, \Omega\right)$ under the identification $\beta \leftrightarrow \Delta_{\beta}$.

Proof. Clearly $\left(G_{\eta}, \leqslant\right)$ is a right ordering. The relation

$$
g_{1} \sim g_{2} \Leftrightarrow \alpha g_{1}=\alpha g_{2}
$$


is a convex congruence $\mathscr{C}$ in the right regular representation of $G_{\eta}$ on the chain $\left(G_{\eta}, \leqslant\right)$, and the action of $G_{\eta}$ on $\mathscr{C}$ coincides with its action on $\Omega$ (identifying $\beta \in \Omega$ with $\Delta_{\beta}$, with the aid of Lemma 1). These two statements apply also to the natural action of $F_{\eta}$ on $\left(G_{\eta}, \leqslant\right)$. For if $g_{1}, g_{2} \in G_{\eta}$, then if $\Delta_{\beta} g_{1}$ meets $\Delta_{\beta} g_{2}$, then $\Delta_{\beta} g_{1}=\Delta_{\beta} g_{2}$. Then for $w \in F_{\eta}, \Delta_{\beta} w=\Delta_{\beta}\left(\bigvee_{i} \wedge_{j} w_{i j}\right)=\max _{i} \min _{j} \Delta_{\beta} w_{i j}=\Delta_{\beta} w_{i^{\prime} j^{\prime}}$ (another $\mathscr{C}$-class) $=\Delta_{\beta w_{i, j},}=\Delta_{\beta w}$. Since $\left(F_{\eta}, \Omega\right)$ is faithful by hypothesis, so is $\left(F_{\eta}, \mathscr{C}\right)$ and thus also $\left(F_{\eta},\left(G_{\eta}, \leqslant\right)\right)$.

The shortest route to Kopytov's representation is to prove just enough of [7, Theorem 1] to get a transitive representation of $F_{\eta}$. This saves only a bit, but for Conrad's representation, there is a much shorter route. Theorem 2 remains valid for transitive actions, the conclusion being that the kernel of the action $\left(F_{\eta},\left(G_{\eta}, \leqslant\right)\right)$ is contained in the kernel of $\left(F_{\eta}, \Omega\right)$. Thus it suffices to note that the prime subgroups $P$ of $F_{\eta}$ afford a collection of transitive actions whose kernels have trivial intersection! ([7, Theorem 1] is completely bypassed.) Of course, Conrad's approach is still needed for the more general notion of an l-group free over a given po-group.

How explicit is the construction in Theorem 2 of the right ordering $\left(G_{\eta}, \leqslant\right)$ and the representation of $F_{\eta}$ thereon? No more explicit than the given transitive representation $\left(F_{\eta}, \Omega\right)$, certainly. But unfortunately, the points $\beta$ of $\Omega$ are replaced by the perhaps complicated $o$-blocks $\Delta_{\beta}$. We shall entirely overcome this second obstacle by arranging about $\left(F_{\eta}, \Omega\right)$ that $\Delta_{\alpha}=F_{\alpha} \cap G_{\eta}$ be $\{e\}$, i.e., that no nonidentity group word fix $\alpha$. Then the natural representation of $F_{\eta}$ on $\left(G_{\eta}, \leqslant\right)$ will coincide with $\left(F_{\eta}, \Omega\right)$. By simultaneously making $\left(F_{\eta}, \Omega\right)$ pathologically $o$-2-transitive, we will get the best of both worlds!

As an extra bonus, we bypass Theorem 2 . The right ordering $\left(G_{\eta}, \leqslant\right)$ becomes simply

$$
g_{1} \leqslant g_{2} \Leftrightarrow \alpha g_{1} \leqslant \alpha g_{2},
$$

and obviously the action $\left(F_{\eta},\left(G_{\eta}, \leqslant\right)\right)$ coincides with the given representation $\left(F_{\eta}, \Omega\right)$ and thus is also a representation.

Incidentally, the preceding argument shows that the transitive representions of $F_{\eta}$ for which no nonidentity group word fixes any point coincide with the natural representations of $F_{\eta}$ on representing right orderings $\left(G_{\eta}, \leqslant\right)$.

MAIN TheORem 3. Let $1<\eta \leqslant \omega_{0}$, or (with G.C.H.) let $\eta$ be regular. Then there exists a right ordering $\left(G_{\eta}, \leqslant\right)$ on which the natural action of $F_{\eta}$ is both faithful and pathologically o-2-transitive. $\left(G_{\eta}, \leqslant\right)$ must be o-isomorphic to $\mathbf{Q}$ if $\eta \leqslant \omega_{0}$, and may be taken to be an $\alpha$-set $\left(\eta=\omega_{\alpha}\right)$ if $\eta$ is regular.

Proof. We shall modify the proof of [7, Theorem 1] so that no $e \neq g \in G$ fixes any point, and Theorem 3 will follow. Having $\beta g=\beta$ ( $g$ a reduced group word $\neq e$ ) would produce a loop, i.e., a nonempty sequence of $x^{ \pm 1}$-arrows (reduced in the obvious sense) such that the head of the last coincides with the tail of the first. Here an $x$-arrow from $\gamma$ to $\delta$ is also thought of as an $x^{-1}$-arrow from $\delta$ to $\gamma$. We want our specifications to be loop-free.

First we treat $\eta \leqslant \omega_{0}$. We retain the specifications made in the first part of the proof of [7, Theorem 1], through the building of all the bridges, and including the 


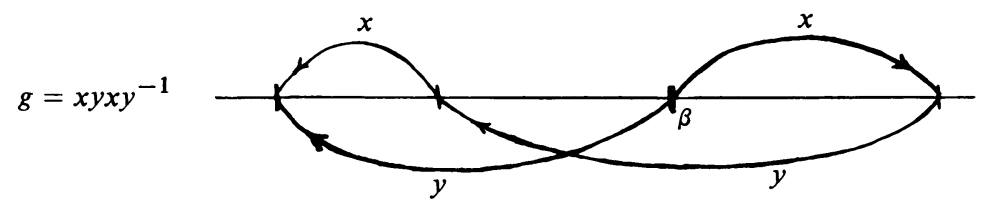

Figure 1. A loop

specification that $(-1) \hat{x}_{r_{0}}^{ \pm 1}=0$, but we change the specifications which guarantee $o$-2-transitivity. For convenience, we change notation: We write $\hat{x}$ as $x$, we let $y=x_{r_{0}}^{ \pm 1}$ (replacing $x_{r_{0}}$ by $x_{r_{0}}^{-1}$ produces another free generating set), and we let $x$ be any other element of $\mathbf{x}$. Also, we negate all the numbers involved in the specifications and then shift them one to the left (see the negative half of the line in Figure 2).

Next we make further specifications as shown in the positive half of the line in Figure 2. Let $q_{1}, q_{2}, \ldots$ be a strictly decreasing sequence of rationals (all less than 1 ), with $q_{n} \downarrow 0$. We specify that:

(a) $1 x=1+q_{1}$

(b) $0 x=1+q_{2}$,

(c) $\left(n+q_{2 n-1}\right) x=n+1+q_{2 n+1}, n \geqslant 1$,

(d) $\left(n+q_{2 n}\right) x=n+1+q_{2 n+2}, n \geqslant 1$,

(e) $\alpha y=\alpha-1$ for all $\alpha \geqslant 1$ which are integers, or which differ by an integer from some $q_{n}$.

Regardless of what further specifications we make, the resulting (transitive) action of $F_{\eta}$ on the orbit $\Omega=0 F_{\eta}$ will be faithful because of the specifications retained from [7]. We claim that $\left(F_{\eta}, \Omega\right)$ will be $o$-primitive. Let $\Delta$ be a nonsingleton $o$-block containing 0 , and thus also containing points above 0 . Specifications (a)-(e) make

$$
0 x^{n} y^{n}=q_{2 n} \text { and } 1 x^{n} y^{n}=q_{2 n+1},
$$

so that

$$
0\left(x^{n} y^{n} \wedge e\right)=0 \text { and } 1\left(x^{n} y^{n} \wedge e\right)=q_{2 n+1} .
$$

Thus the elements $x^{n} y^{n} \wedge e$ fix $\Delta$ and move 1 down arbitrarily close to 0 , making $\Delta$ contain 1. Since $n y^{-1}=n+1(n \geqslant 0), \Delta$ must be cofinal in $\Omega$, so that $\Delta=\Omega$. Therefore $\left(F_{\eta}, \Omega\right)$ is $o$-primitive. But every $o$-primitive representation of $F_{\eta}$ is pathologically $o$-2-transitive [7, Proposition 13].

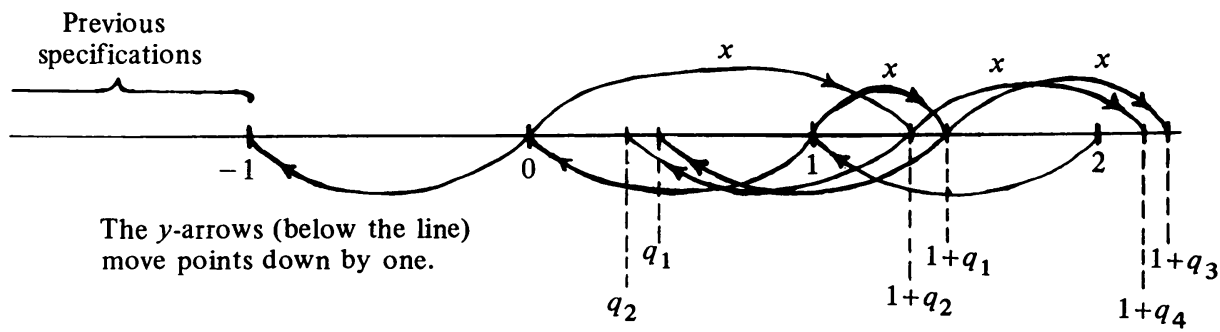

FIGURE 2 
So far we have created no loops: There are none in the specifications retained from the proof of [7, Theorem 1] because diagrams are loop-free, and none are created by (a)-(e) because the $q_{n}$ 's are strictly decreasing. To finish specifying the free generators without accidently forming any loops, we enumerate the set of ordered pairs $(\alpha, z)$, where $\alpha \in \mathbf{Q}$ and $z$ is a free generator or the inverse of one. Proceeding inductively, we specify a $z$-arrow with tail at $\alpha$ (unless one has already been specified, either earlier in the induction or prior to the induction).

The only limit points (rational or irrational) of the specified points are the nonnegative integers; and if the $y$-arrows are deleted, there are no limit points at all. Thus of the tails of $z$-arrows below $\alpha$, let $\beta_{L}$ be the largest $\left(\beta_{L}=-\infty\right.$ if there are no such tails); and of the tails of $z$-arrows above $\alpha$, let $\beta_{U}$ be the smallest ( $\beta_{U}=+\infty$ if there are no such tails). This makes sense unless $z=y$ and $1 \leqslant \alpha \in \mathbf{Z}$ or $z=y^{-1}$ and $0 \leqslant \alpha \in \mathbf{Z}$, and in these cases a $z$-arrow with tail at $\alpha$ has already been specified. As the head of the $z$-arrow with tail at $\alpha$, we choose any point $\delta \neq \alpha$ which has not previously been specified (as an end of any arrow at all), and which is greater than the head $\beta_{L}^{\prime}$ of the $z$-arrow with tail at $\beta_{L}$ (no restriction here if $\left.\beta_{I .}=-\infty\right)$ and less than the head $\beta_{U}^{\prime}$ of the $z$-arrow with tail at $\beta_{U}$. The above remark about limit points guarantees the existence of such a $\delta$, and the choice of $\delta$ preserves consistency of $z$-arrows.

For each $z \in \mathbf{x}$, this construction specifies an $o$-automorphism $z$ of $\mathbf{Q}$ (onto $\mathbf{Q}$ because of the inclusion of $z^{-1}$ in the induction). There were no loops before we began the induction, and the induction cannot produce a loop because the last arrow, which completed the loop, would have had as its head an already specified point, contrary to the above choice of $\delta$. Restricting to $\Omega=0 F_{\eta}$, we complete the construction for $\eta=\omega_{0}$. Of course, in any $o$-2-transitive representation $\left(F_{\eta}, \Omega\right), \Omega$ must be countable and dense in itself, and thus $o$-isomorphic to $\mathbf{Q}$.

Now let $\eta$ be regular. Write $\eta$ as $\omega_{\alpha}$, and in the above argument, replace $\mathbf{Q}$ by an $\alpha$-set $\Lambda$ [3, p. 187], using the G.C.H. (For $\eta=\omega_{0}$, the G.C.H. is not needed. Alternately, the previous argument can be applied.) Select a set $\mathscr{D}$ of pairwise disjoint open intervals $\Delta$ of $\Lambda$ whose union is coinitial in $\Lambda$, and such that the order type of $\mathscr{D}$ is the reverse of the ordinal number $\omega_{\alpha}$. Select a one-to-one correspondence between the set of nonidentity elements of $F_{\eta}$ and the set $\mathscr{D}$, and for each $e \neq w \in F_{\eta}$ specify within the corresponding $\Delta$ a diagram showing $e \neq w$. Since some $\Delta$ 's lack immediate successors, the bridging must be modified. Let $\Delta_{0}$ be the greatest $\Delta \in \mathscr{D}$. For each $\Delta_{0} \neq \Delta \in \mathscr{D}$, pick $x_{\Delta} \in \mathbf{x}$ which does not appear in the diagram for any $\Delta \geqslant \Delta^{\prime} \in \mathscr{D}$ (by the regularity of $\omega_{\alpha}$, fewer than $\omega_{\alpha} x$ 's so appear)

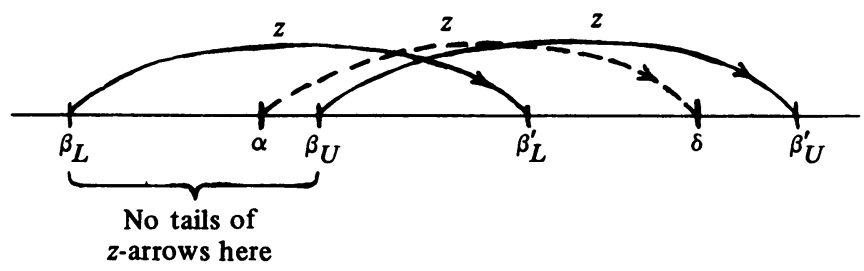

Figure 3 
and make the $x_{\Delta}$ 's distinct. Form an $x_{\Delta}$-arrow from any point of $\Delta_{0}$ to any point of $\Delta$. Let $y$ be a free generator moving up the greatest point $\rho$ in the diagram within $\Delta_{0}$ (replace $y$ by $y^{-1}$ if necessary). We specify a $y$-arrow moving $\rho$ up to some point artificially denoted by “ 0 ".

Instead of (a)-(e), we proceed as follows: Within $\Lambda^{+}=\{\lambda \in \Lambda \mid \lambda>0\}$, pick a descending copy $\Lambda_{D}$ of the ordinal number $\omega_{\alpha}$ having inf 0 , and entirely above it an ascending copy $\Lambda_{A}$ of $\omega_{\alpha}$ having no upper bound. Select a one-to-one correspondence between $\Lambda_{A}$ and $\mathbf{x}$. For each $\lambda \in \Lambda_{A}$, specify that the corresponding $x_{\lambda}$ move it down to the corresponding point $\lambda^{\prime}$ of $\Lambda_{D}$, and move 0 up to any point between $\lambda^{\prime}$ and the next largest point of $\Lambda_{D}$. Then $x_{\lambda} \wedge e$ will fix 0 and move $\lambda$ to $\lambda^{\prime}$, which will guarantee the $o$-primitivity of the representation (whether or not $\lambda, \lambda^{\prime} \in 0 F_{\eta}$ ), and thus the pathological $o$-2-transitivity.

"Enumerate" as in the finite case, using $\omega_{\alpha}$ instead of $\omega_{0}$. When specifying a $z$-arrow with tail at $\alpha$, let $\beta_{L}^{\prime}$ be the sup in $\bar{\Lambda}$ of the heads of $z$-arrows whose tails lie below $\alpha$, and $\beta_{U}^{\prime}$ its dual. Since $\beta_{L}^{\prime}$ is the sup of a set of cardinality less than $\omega_{\alpha}$ (by regularity), and $\beta_{U}^{\prime}$ dually, the properties of $\alpha$-sets guarantee that there exist points of $\Lambda$ between $\beta_{L}^{\prime}$ and $\beta_{U}^{\prime}$. Again by regularity and the properties of $\alpha$-sets, no point of $\Lambda$ except 0 is a limit point of already specified points (although there may be other limit points in $\bar{\Lambda}$ ), as we can choose a head for the $z$-arrow which preserves consistency.

Since $\left(G_{\eta}, \leqslant\right)$ is $o$-isomorphic to $\Lambda$, this concludes the proof of Theorem 3 .

Admittedly the right $\operatorname{order}\left(G_{\eta}, \leqslant\right)$ obtained from the representation $\left(F_{\eta}, \Omega\right)$ of Theorem 3 by setting

$$
g_{1} \leqslant g_{2} \Leftrightarrow \alpha g_{1} \leqslant \alpha g_{2}
$$

is far from being explicit. However, the rest of this paper is devoted to the idea that we have enough control over the representation to learn a great deal about prime subgroups of $F_{\eta}$.

3. The root system of prime subgroups of $F_{\eta}$. Let $\mathscr{P}_{\eta}$ denote the root system of prime subgroups of $F_{\eta}$. Here we include $\eta=1$ and $\eta=0 . F_{1}=\mathbf{Z} \boxplus \mathbf{Z}$, so $\mathscr{P}_{1}$ has three elements, two minimal and one lying above them. $F_{0}=\{e\}$, so $\mathscr{P}_{0}$ is singleton.

$\mathscr{P}_{\eta}$ has $F_{\eta}$ as its largest element. Its branches are the connected components of $\mathscr{P}_{\eta} \backslash\left\{F_{\eta}\right\}$. For $P \in \mathscr{P}_{\eta}, \mathscr{L}(P)$ will denote $\left\{Q \in \mathscr{P}_{\eta} \mid Q \leqslant P\right\}$.

The roots of $\mathscr{P}_{\eta}$ are the maximal subchains. Within each root the set of covering pairs is dense, and the bottom halves of these covering pairs are the values within that root. Given that for finite $\eta$ every branch of $\mathscr{P}_{\eta}$ has a largest element [7, Corollary 16], we shall find that every conceivable chain occurs as the chain of covering pairs (equivalently, of values) within some root of $\mathscr{P}_{\eta}$.

Let $\mathscr{R}_{\eta} \subseteq \mathscr{P}_{\eta}$ denote the root system of representing subgroups of $F_{\eta}$, together with $F_{\eta}$ itself. (For finite $\eta$, it was not known until [7] that $F_{\eta}$ even has any representing subgroups, i.e., any transitive representations.) [7, Corollary 16] established that for finite $\eta$, every branch of $\mathscr{P}_{\eta}$ containing any representing subgroups at all consists entirely of representing subgroups, so that $\mathscr{R}_{\eta}$ consists of some set of entire branches of $\mathscr{P}_{\eta}$ (together with $F_{\eta}$ at the top). In fact, $\mathscr{R}_{\eta}$ is exceedingly much like $\mathscr{P}_{\eta}$. 
MAIN TheOREM 4A. For finite $\eta>1$, all the root systems $\mathscr{P}_{\eta}$ are o-isomorphic to each other, and also to the root systems $\mathscr{R}_{\eta}$. For this common root system $\mathscr{P}_{f}$ :

(1) $\operatorname{Card}\left(\mathscr{P}_{f}\right)=2^{\omega_{0}}$.

(2) Each branch contains a (unique) largest element.

(3) Every branch is o-isomorphic to $\mathscr{P}_{0}, \mathscr{P}_{1}, \mathscr{P}_{f}$, or $\mathscr{P}_{\omega_{0}}$ (no two of which are $o$-isomorphic); and there are $2^{\omega_{0}}$ branches of each type.

(4) For $P \in \mathscr{P}_{f}, \mathscr{L}(P)$ is o-isomorphic to $\mathscr{P}_{0}, \mathscr{P}_{1}, \mathscr{P}_{f}$, or $\mathscr{P}_{\omega_{0}}$.

(5) The chains of covering pairs in the roots of $\mathscr{P}_{f}$ are precisely the finite and countable chains having largest elements.

MAIN THEOREM 4B. For $\mathscr{P}_{\omega_{0}}$ :

(1) $\operatorname{Card}\left(\mathscr{P}_{\omega_{0}}\right)=2^{\omega_{0}}$.

(2) The isomorphism types of the branches are $\mathscr{P}_{0}, \mathscr{P}_{1}, \mathscr{P}_{f}, \mathscr{P}_{\omega_{0}}$ and $2^{\omega_{0}}$ types (each of cardinality $2^{\omega_{0}}$ ) having no largest element. Each of these types occurs $2^{\omega_{0}}$ times.

(3) For each $P \in \mathscr{P}_{\omega_{0}}, \mathscr{L}(P)$ is o-isomorphic to $\mathscr{P}_{0}, \mathscr{P}_{1}, \mathscr{P}_{f}$, or $\mathscr{P}_{\omega_{0}}$.

(4) The chains of covering pairs in the roots of $\mathscr{P}_{\omega_{0}}$ are precisely the nonempty finite and countable chains.

(5) The above statements apply verbatim to $\mathscr{R}_{\omega_{0}}$ (the lists in (3) and (4) still being $\mathscr{P}_{0}, \mathscr{P}_{1}, \mathscr{P}_{f}, \mathscr{P}_{\omega_{0}}$ rather than $\left.\mathscr{R}_{0}, \mathscr{R}_{1}, \mathscr{R}_{f}, \mathscr{R}_{\omega_{0}}\right)$.

MAIN THEOREM 4C. Let $\eta>\omega_{0}$ be regular.

(1) $\operatorname{Card}\left(\mathscr{P}_{\eta}\right)=2^{\eta}$.

(2) The o-isomorphism types of the branches of $\mathscr{P}_{\eta}$ are (a) $\mathscr{P}_{0}, \mathscr{P}_{1}, \mathscr{P}_{f}$ and $\mathscr{P}_{\mu}$ $\left(\omega_{0} \leqslant \mu \leqslant \eta\right.$ ), (b) $2^{\eta}$ types of cardinality $2^{\eta}$ and cofinality $\mu$ (for each regular $\mu$ such that $\omega_{0} \leqslant \mu \leqslant \eta$ ), (c) perhaps some types of cardinality $\eta$ and cofinality $\eta$. Each type occurs $2^{\eta}$ times, except perhaps for the types in (b) with $\mu<\eta$, and even these occur at least $\eta$ times.

(3) For each $P \in \mathscr{P}_{\eta}, \mathscr{L}(P)$ is o-isomorphic to $\mathscr{P}_{0}, \mathscr{P}_{1}, \mathscr{P}_{f}$, or $\mathscr{P}_{\mu}\left(\omega_{0} \leqslant \mu \leqslant \eta\right)$.

(4) Every chain of cardinality at most $\eta$ occurs as an upper ray of the chain of covering pairs of some root of $\mathscr{P}_{\eta}$.

(5) The above statements apply verbatim to $\mathscr{R}_{\eta}$.

We need many a lemma. The first one generalizes certain aspects of Theorem 2. Let $H$ be a (not necessarily normal) subgroup of $G_{\eta}$. By a right ordering of $G_{\eta} / H$ we shall mean a total order $\left(G_{\eta} / H, \leqslant\right)$ of the set $G_{\eta} / H$ of right cosets $\mathrm{Hg}\left(g \in G_{\eta}\right)$ which is preserved by right multiplication by elements of $G_{\eta}$. (When $H=\{e\}$, this is

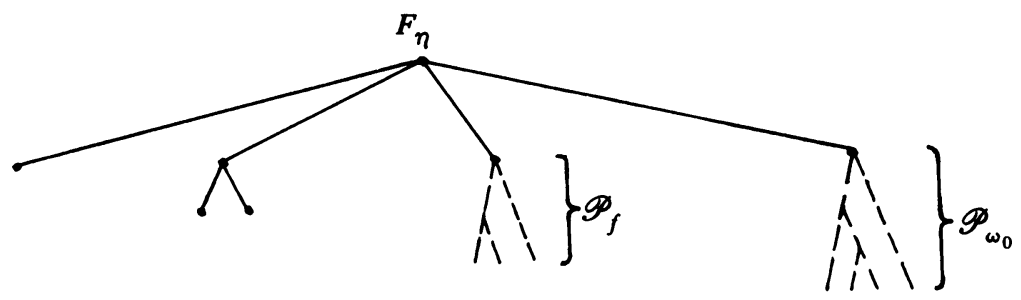

FIGURE 4. The branches of $\mathscr{P}_{f}$, each of which occurs $2^{\omega_{0}}$ times 
the usual definition of a right ordering of $G_{\eta}$.) The natural action of $F_{\eta}$ on $\left(G_{\eta} / H, \leqslant\right)$ will mean the unique extension of the action of $G_{\eta}$ to an action of $F_{\eta}$.

For $K$ a subgroup of $F_{\eta}$, we denote by $l(K)$ the $l$-subgroup generated by $K$.

Lemma 5. Let $P$ be a prime subgroup of $F_{\eta}$, and $H$ a subgroup of $P \cap G_{\eta}$. Then given any right ordering $\left(\left(P \cap G_{\eta}\right) / H, \preccurlyeq\right)$, there exists a unique right ordering $\left(G_{\eta} / H, \leqslant\right)$ for which

(a) The sets $\left(\left(P \cap G_{\eta}\right) g\right) / H$ are the classes of a convex congruence $\mathscr{C}$ in the natural action of $F_{\eta}$ on $\left(G_{\eta} / H, \leqslant\right)$.

(b) The action of $F_{\eta}$ on $\mathscr{C}$ coincides with its action on $F_{\eta} / P$ (under the identification $\left.\left(\left(P \cap G_{\eta}\right) / H \leftrightarrow P g\right)\right)$.

(c) The action of $l\left(P \cap G_{\eta}\right)$ on $\left(\left(P \cap G_{\eta}\right) / H, \leqslant\right)$ coincides with its action on $\left(\left(P \cap G_{\eta}\right) / H, \preccurlyeq\right)$.

PROOF. The only candidate for such a right ordering of $G_{\eta} / H$ is

$$
\begin{gathered}
H g_{1} \leqslant H g_{2} \Leftrightarrow \\
\left(P g_{1}<P g_{2} \text { in } F_{\eta} / P \text { or else, } P g_{1}=P g_{2} \text { and } H \preccurlyeq H g_{2} g_{1}^{-1} \text { in }\left(P \cap G_{\eta}\right) / H\right) \text {. }
\end{gathered}
$$

This order is well defined. For if $H g_{1}=H \bar{g}_{1}$ and $H \preccurlyeq H g_{2} g_{1}^{-1}$, then $H g_{1} \bar{g}_{1}^{-1} \preccurlyeq$ $\left(H g_{2} g_{1}^{-1}\right) g_{1} \bar{g}_{1}^{-1}$ since the curly order is preserved under right multiplication by $H$, i.e., $H \preccurlyeq H g_{2} \bar{g}_{1}^{-1}$; and the rest is clear. We have a right ordering satisfying (a). By Lemma $1, G_{\eta}$ acts transitively on $F_{\eta} / P$, justifying the identification in (b). Now the actions in (b) coincide for $G_{\eta}$ and thus also for $F_{\eta}$. The same holds in (c) for $P \cap G_{\eta}$ and thus also for $l\left(P \cap G_{\eta}\right)$, given that the two orderings of $\left(P \cap G_{\eta}\right) / H$ coincide because the action of $P \cap G_{\eta}$ preserves the curly order.

Lemma 6. Let $P$ be a prime subgroup of $F_{\eta}$. Then $l\left(P \cap G_{\eta}\right)$ is a free l-group, with $P \cap G_{\eta}$ the subgroup ( freely) generated by some free generating set $\mathbf{x}^{\prime}$ of $l\left(P \cap G_{\eta}\right)$. Thus l-group $\operatorname{rank}\left(l\left(P \cap G_{\eta}\right)\right)=\operatorname{group} \operatorname{rank}\left(P \cap G_{\eta}\right)$.

Proof. In view of Lemma 5, this follows from [2, Theorem 3.9]. However, we give here a proof in the spirit of our other results.

Pick a free generating set $\mathbf{x}^{\prime}$ for the free group $P \cap G_{\eta}$, whose rank we denote by $\mu$. If $\mu=0$, the lemma is trivial. If $\mu=1$, the free generator $x$ is incomparable with $e$ in $F_{\eta}$ (this is true of all nonidentity elements of $G_{\eta}$ ) and thus freely generates $l\left(P \cap G_{\eta}\right)$. Accordingly we suppose $\mu>1$.

We want to show that every $l$-group word $w$ in $\mathbf{x}^{\prime}$ which is not the identity in the free $l$-group $F_{\eta}$ on $\mathbf{x}^{\prime}$ is not the identity even when the sups and infs are taken in the given $F_{\eta}$. By Theorem 2 (since $\mu>1$ ), $w \varphi \neq e$ in the natural representation $\varphi$ of $F_{\eta}$ on some right ordering ( $\left.P \cap G_{\eta}, \preccurlyeq\right)$. We apply Lemma 5 to extend $\left(P \cap G_{\eta}, \preccurlyeq\right)$ to a right ordering $\left(G_{\eta}, \leqslant\right)$ on which the natural action $\psi$ of $F_{\eta}$ has $P \cap G_{\eta}$ as an $o$-block. For $h \in P \cap G_{\eta}$ and thus also for $h \in l\left(P \cap G_{\eta}\right)$, the restriction $(h \psi) \mid\left(P \cap G_{\eta}\right)=$ $h \varphi$. Since $w \varphi \neq e$, we have $w \psi \neq e$ and thus $w \neq e$ in $F_{\eta}$.

LeMma 7. Let $P$ be a prime subgroup of $F_{\eta}$. Then the root system $\mathscr{L}(P)$ is $o$-isomorphic to the root system of all prime subgroups of $l\left(P \cap G_{\eta}\right)$; and thus to $\mathscr{P}_{\mu}$, where $\mu=l$-group $\operatorname{rank}\left(l\left(P \cap G_{\eta}\right)\right)=\operatorname{group} \operatorname{rank}\left(P \cap G_{\eta}\right)$. 
Proof. Denote the two root systems by $\mathscr{L}$ and $\mathscr{T}$, respectively. The map $Q \rightarrow Q$ $\cap l\left(P \cap G_{\eta}\right)$ sends $\mathscr{L}$ into $\mathscr{T}$ and preserves order. In the other direction, let $Q^{\prime} \in \mathscr{T}$. Right order $\left(P \cap G_{\eta}\right) /\left(Q^{\prime} \cap G_{\eta}\right)$ according to its identification with the chain $l\left(P \cap G_{\eta}\right) / Q^{\prime} \quad$ (i.e., $\left(Q^{\prime} \cap G_{\eta}\right) g \leftrightarrow Q^{\prime} g$, which works because for $h \in$ $l\left(P \cap G_{\eta}\right)$,

$$
Q^{\prime} h=Q^{\prime}\left(\bigvee_{i} \bigwedge_{j} g_{i j}\right)\left[g_{i j} \in P \cap G_{\eta}\right]=\bigvee_{i} \bigwedge_{j} Q^{\prime} g_{i j}=Q^{\prime} g_{i^{\prime} j^{\prime}}
$$

for some $\left.g_{i^{\prime} j^{\prime}}\right)$. Extend this right order to a right order $\left(G_{\eta} /\left(Q^{\prime} \cap G_{\eta}\right), \leqslant\right)$ in the manner afforded by Lemma 5 (with $H=Q^{\prime} \cap G_{\eta}$ ). In the natural action $\varphi$ of $F_{\eta}$ on $\left(G_{\eta} /\left(Q^{\prime} \cap G_{\eta}\right), \leqslant\right)$, let $Q^{\prime \prime}$ be the stabilizer of the point $Q^{\prime} \cap G_{\eta}$. By (a) and (b) of Lemma $5, \Delta=\left(P \cap G_{\eta}\right) /\left(Q^{\prime} \cap G_{\eta}\right)$ is an $o$-block of this action, and since the action coincides with the action of $F_{\eta}$ on $F_{\eta} / P$ (with $\Delta \leftrightarrow P$ ), the stabilizer of $\Delta$ is $P$. Since the point $Q^{\prime} \cap G_{\eta}$ lies in $\Delta, Q^{\prime \prime} \subseteq P$. This makes $Q^{\prime \prime} \in \mathscr{S}$. We map $Q^{\prime} \rightarrow Q^{\prime \prime}$. This map from $\mathscr{T}$ to $\mathscr{L}$ preserves order for the same reason that $Q^{\prime \prime} \subseteq P$.

Let $Q \in \mathscr{L}$, and let $Q^{\prime}=Q \cap l\left(P \cap G_{\eta}\right)$. The right ordering $\left(G_{\eta} /\left(Q^{\prime} \cap G_{\eta}\right), \leqslant\right)$ used to form $Q^{\prime \prime}$ coincides with chain $F_{\eta} / Q$ (via $\left(Q^{\prime} \cap G_{\eta}\right) g \leftrightarrow Q g$ ). This is true by construction within $\left(P \cap G_{\eta}\right) /\left(Q^{\prime} \cap G_{\eta}\right)$, where $\left(Q^{\prime} \cap G_{\eta}\right) g \leftrightarrow Q^{\prime} g \leftrightarrow Q g$ (an arbitrary element of $P / Q$ ). Elsewhere it follows from Lemma 5 (part (b) and the definition of the right ordering). Hence the stabilizer $Q^{\prime \prime}$ of the point $Q^{\prime} \cap G_{\eta}$ is $Q$, as desired.

Each $Q^{\prime} \in \mathscr{T}$ is the stabilizer of the point $Q^{\prime}$ in the action of $l\left(P \cap G_{\eta}\right)$ on $l\left(P \cap G_{\eta}\right) / Q^{\prime}$. By (c) of Lemma 5, $Q^{\prime} \supseteq Q^{\prime \prime} \cap l\left(P \cap G_{\eta}\right) \supseteq Q^{\prime}$, making $Q^{\prime \prime} \cap$ $l\left(P \cap G_{\eta}\right)=Q^{\prime}$. Therefore our two mappings are inverses of each other.

LEMMA 8. A prime subgroup $P$ of $F_{\eta}$ is minimal if and only if $P \cap G_{\eta}=\{e\}$.

Proof. $P$ is minimal iff $\mathscr{L}(P)=P$ iff group $\operatorname{rank}\left(P \cap G_{\eta}\right)=0$, by Lemma 7 .

LeMMA 9A. Let $1<\eta \leqslant \omega_{0}$. For each $0 \leqslant \mu \leqslant \omega_{0}, F_{\eta}$ has precisely $2^{\omega_{0}}$ representing maximal prime subgroups $P$ for which $l\left(P \cap G_{\eta}\right)$ is a free l-group of rank $\mu$. For each such $P$, the representation $\left(F_{\eta}, F_{\eta} / P\right)$ is pathologically o-2-transitive.

REMARK. This establishes that $G_{\eta}$ has $2^{\omega_{0}}$ representing right orderings.

Proof. For the necessity that $\left(F_{\eta}, F_{\eta} / P\right)$ be pathologically $o$-2-transitive, see [7, Proposition 13]. Clearly the number of such $P$ 's is no more than stated. We construct the desired $P$ 's by varying the proof of Theorem 3 .

First we consider the case $\eta=2$ and $\mu=\omega_{0}$ and construct a single $P$. Let $x$ and $y$ be the free generators of $F=F_{2}$, and let $h_{p}=x^{p} y^{p} x^{-p} y^{-p}(p \geqslant 1)$. The $h_{p}$ 's freely generate a subgroup $H$ (of rank $\omega_{0}$ ) of $G_{2}$. (For any reduced group word in the $h_{p}$ 's the number of alternations between powers of $x$ and powers of $y$ increases as each additional generator is used in forming that group word.) Pick an irrational number $r$ slightly greater than 1 . We begin with the same specifications from the proof of [7, Theorem 1] that were used in the proof of Theorem 3, modified in the same way; except that we make $(-1) y=0$ instead of vice versa, and we replace $\mathbf{Q}$ by the subchain $\mathbf{Q} \oplus \mathbf{Q} r$ of $\mathbf{R}$, which of course is also countable and dense in itself. 
To make the stabilizer $F_{0}$ have the properties desired of $P$, we make additional specifications to force $F_{0} \cap G_{\eta}=H$. For (a)-(d), $x$ is to move each specified point up by 1 , and $y$ is to move each specified point up by $r$. For each $n \in \mathbf{Z}^{+}$, we specify:

(a) $x$ at each $m=0, \ldots, n-1$,

(b) $y$ at each $n+m r, m=0, \ldots, n-1$,

(c) $x^{-1}$ at each $n+n r-m, m=0, \ldots, n-1$,

(d) $y^{-1}$ at each $n+n r-n-m r=(n-m) r, m=0, \ldots, n-1$.

For $n=1$, this is illustrated in Figure 5. The (a)'s for the various $n$ 's specify $x$ (with considerable redundancy) at every $n \geqslant 0$; and the (d)'s specify $y$ at every $n r, n \geqslant 0$. Since $r$ is irrational, no point except the $n$ 's and $n r$ 's is involved in more than one of these specifications.

For each $h_{p}$, we have produced a loop beginning at 0 . This will make $H \subseteq F_{0} \cap G_{\eta}$. As further specifications are made later in the proof, we shall arrange that

No arrow ever reconnects two points already connected by some pre-existing sequence of arrows.

We claim that this will force every reduced group word $g \in F_{0} \cap G_{\eta}$ to be also a group word in the $h_{p}$ 's, so that $H=F_{0} \cap G_{\eta}$.

Suppose it turns out that $g$ gives a sequence of arrows beginning at 0 and returning to 0 . Since there are no loops in the specifications carried over from the proof of [7, Theorem 1], (*) guarantees that $g$ begins with $x$ or $y$ rather than $x^{-1}$ or $y^{-1}$. Otherwise, some sequence of arrows given by a reduced group word and beginning with an $x^{-1}$ - or $y^{-1}$-arrow at 0 would return to 0 . Then the arrow in this sequence which was specified last would have reconnected two already connected points, violating (*). We suppose the former, the latter case being similar. Thus $g=x^{q} y^{ \pm 1} \ldots$ for some $q \geqslant 1$. But if we had $y^{-1}$, the fact that $y^{-1}$ is not specified at $q$ by any (b) or (d), together with (*) and the fact that $g$ is reduced, would make it impossible ever to return to 0 . Thus $g=x^{q} y \cdots$. Similarly, the next several letters must be $y$ 's, so that $g=x^{q} y^{q} \ldots$, and then the next several must be $x^{-1}$ 's, so that $g=x^{q} y^{q} x^{-q} \ldots=x^{q} y^{q} x^{-q} k$ for some $k \in G_{\eta}$. Finally there are two possibilities: $y^{ \pm 1}$. However, we write $g=x^{q} y^{q} x^{-q} y^{-q} y^{q} k=h_{q} y^{q} k$. We have $y^{q} k \in F_{0} \cap G_{\eta}$, and by induction on the length of $g, y^{q} k \in H$, making $g \in H$ and proving the claim.

In order to make the powers of $h_{1}=x y x^{-1} y^{-1}$ move 1 down arbitrarily close to 0 (which they leave fixed), we make some further specifications (with $y$ still moving all specified points up by $r$, but with $x$ moving points up by various amounts). We specify that

(e) $x^{-1}$ move $3+r$ down by more than one (but not move it past 2 , to maintain consistency with the previous $x$-arrows). Letting $\beta_{1}=1$, this makes $\beta_{2}=\beta_{1} h_{1}=$ $\beta_{1} x y x^{-1} y^{-1}=(3+r) x^{-1} y^{-1}<(2+r) y^{-1}=1=\beta_{1}$.

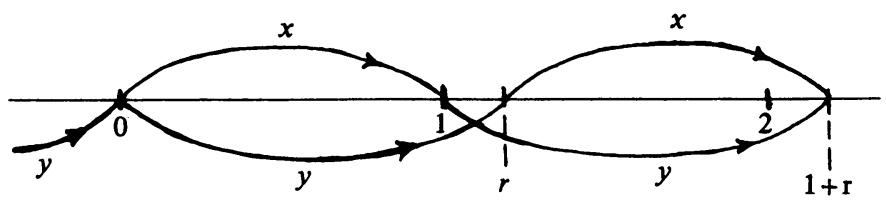

FIGURE 5 
(f) $x$ move $\beta_{i}(i=2,3, \ldots)$ up between 1 and $1+1 / i$ (arranging that $\left.\beta_{i} x<\beta_{i-1} x\right)$, and $x^{-1}$ move $\beta_{i} x y$ down by 1 , so that $\beta_{i+1}=\beta_{i} h_{1}=\beta_{i} x y x^{-1} y^{-1}<(1+1 / i)+r-$ $1-r=1 / i$; except that if $\beta_{i} x, \beta_{i} x y, \beta_{i} x y x^{-1}$, or $\beta_{i} x y x^{-1} y^{-1}$ should happen to coincide with one of the finitely many already specified points for which this is possible, $\beta_{i} x$ is to be decreased slightly so that this does not happen.

As in the proof of Theorem 3, regardless of what further specifications we make, the resulting action of $F$ on the orbit $\Omega=0 F$ will be faithful. If $\Delta$ is a nonsingleton $o$-block containing 0 and thus also points above 0 , the fact that the powers of $h_{1}$ move 1 down arbitrarily close to 0 will force $\Delta$ to contain 1 , and the fact that $n x=n+1(n \geqslant 0)$ will then force $\Delta=\Omega$. Hence $(F, \Omega)$ will be $o$-primitive and thus pathologically $o$-2-transitive. This will make the representing subgroup $F_{0}$ a maximal prime subgroup of $F[3$, Theorem 4.1.5]. We complete the specification of $x$ and $y$ via an enumeration like that used in the proof of Theorem 3, and (*) holds. By Lemma $6, l\left(F_{0} \cap G_{\eta}\right)$ is a free l-group whose

$$
\operatorname{rank} \mu=\operatorname{group} \operatorname{rank}\left(F_{0} \cap G_{\eta}\right)=\operatorname{group} \operatorname{rank}(H)=\omega_{0} .
$$

For $\eta=2$ and $\mu=\omega_{0}$, we have produced a single $P\left(=F_{0}\right)$ of the kind desired. We need some more. We partition $\mathbf{Z}^{+}$into three infinite sets $A \cup B \cup C$, with $1 \in A$. For $n \in A$, we specify (a)-(d) exactly as before. For $n \in B \cup C$, we specify (a)-(d) as before with two exceptions. We specify

(c') in (c), for $m=n-1$ only, that $x^{-1}$ move $n+n r-m=n r+1$ down by $1+q_{n}$ if $n \in B$ (but by $1-q_{n}$ if $n \in C$ ). Here $q_{n}$ is a small positive rational number, sufficiently small that this specification is consistent with all the finitely many already existing $x^{-1}$-arrows with which it could conflict, and chosen so that $q_{n} \downarrow 0$ to avoid creating new limit points for the set of specified points.

(d') $y^{-1}$ move down by $r$ each $(n-m) r \mp q_{n}, m=0, \ldots, n-1$. The proof is completed as before. $F_{0} \cap G_{\eta}$ is freely generated by the infinite set $\left\{h_{p} \mid p \in A\right\}$. Moreover, $P=F_{0}$ contains $h_{p} \vee e$ but not $h_{p} \wedge e$ when $p \in B$ (and vice versa when $p \in C)$.

We have produced $2^{\omega_{0}} P$ 's of the desired kind, completing the proof for the case $\eta=2, \mu=\omega_{0}$.

All the other cases are similar for all $\eta$ and all $\mu \neq 0$. In the specifications beyond those carried over from the proof of [7, Theorem 1], all generators except two should be ignored until the enumeration, and the set $A$ should have cardinality $\mu$.

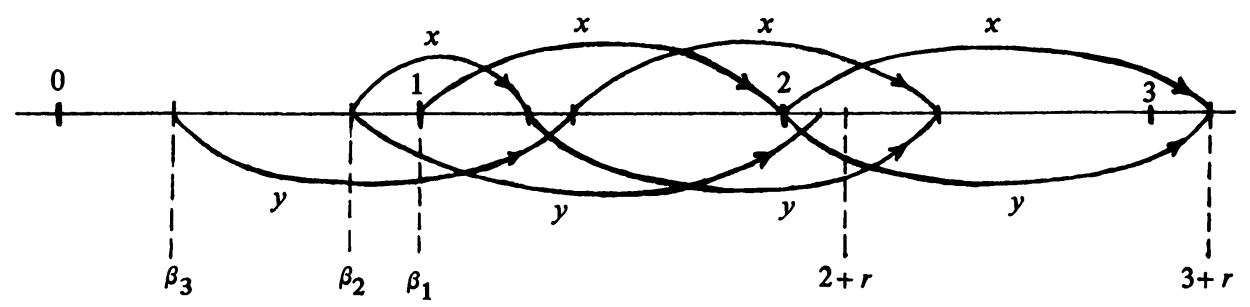

Figure 6 
For $\mu=0$ (and any $\eta$ ), Theorem 3 provides one $P$ of the desired kind. Now we make it provide $2^{\omega_{0}}$ of them. Partition $\{2,3, \ldots\}$ as $B \cup C$. In the proof of Theorem 3 , specify further that for $n \geqslant 2$

(f) $n x=n+1 \pm \frac{1}{2}\left(q_{2 n-1}+q_{2 n}\right)$, choosing " + " if $n \in B$ and "- " if $n \in C$,

(e') $\alpha y=\alpha-1$ for all $\alpha \geqslant 1$ which differ by an integer from some point $n+1$ $\pm \frac{1}{2}\left(q_{2 n-1}+q_{2 n}\right)$. Then for $n \in B$, the stabilizer $P=F_{0}$ contains $y^{-n} x y^{n+1} \wedge e$ but not $y^{-n} x y^{n+1} \vee e$ (and vice versa for $n \in C$ ).

Lemma 9B. Let $\eta$ be regular (G.C.H.). For each $0 \leqslant \mu \leqslant \eta, F_{\eta}$ has precisely $2^{\eta}$ representing maximal prime subgroups $P$ for which $l\left(P \cap G_{\eta}\right)$ is free of rank $\mu$. For each such $P$, the representation $\left(F_{\eta}, F_{\eta} / P\right)$ is pathologically o-2-transitive.

Proof. Again we modify the proof of Theorem 3. Partition $\mathbf{x} \backslash\{y\}$ as $A \cup B \cup$ $C \cup D$, with $\operatorname{card}(A)=\mu$ and $\operatorname{card}(B)=\operatorname{card}(C)=\operatorname{card}(D)=\eta$. Use $D$ as $\mathbf{x}$ was used in the proof of Theorem 3 to map elements of $\Lambda_{A}$ to elements of $\Lambda_{D}$. Specify that $0 x=0$ when $x \in A, 0 x>0$ when $x \in B$, and $0 x<0$ when $x \in C$. Complete the proof as before. Then for $P=F_{0}$, we have $P \cap G_{\eta}$ freely generated by $A$, with $x \wedge e \in P$ but $x \vee e \notin P$ when $x \in B$ (and vice versa when $x \in C$ ).

LEMMA 10. For every nonempty finite or countable chain $\Gamma, F_{\omega_{0}}$ has a transitive representation in which the tower of covering pairs of convex congruences is o-isomorphic to $\Gamma$, and the point stabilizers are minimal prime subgroups.

Proof. We blend the proofs of [7, Theorem 17] and Theorem 3, with the goal of making the stabilizer $F_{0} \cap G_{\omega_{0}}=\{e\}$, where $F=F_{\omega_{0}}$. We begin as in [7], and use the notation developed there. Here $\Lambda=\mathbf{Q}$, so $\Omega$ is countable and dense in itself.

First consider the case in which $\Gamma$ has no largest element. Enumerate the nonidentity elements of $F: w_{1}, w_{2}, \ldots$ Pick an ascending cofinal sequence $\gamma_{1}, \gamma_{2}, \ldots$ in $\Gamma$, indexed by $\mathbf{Z}^{+}$. Lay out in $\mathbf{Q}$ a diagram showing $w_{n} \neq e$, with smallest point $\sigma$ and largest point $\tau$ ( $\sigma$ and $\tau$ independent of $n$ ), with $0<\sigma<\tau$. Using these elements of $A(\mathbf{Q})$ for the $o$-primitive components $x_{\gamma_{n} .0}$, and taking all smaller $o$-primitive components which are involved to be the identity, lay out in $\Omega$ a diagram $\mathscr{D}_{n}$ showing $w_{n} \neq e$, with smallest point $\sigma_{\gamma_{n}}$ and largest point $\tau_{\gamma_{n}}$ (cf. [7]).

Enumerate $\mathbf{x}_{1}$. Proceeding by induction on $n$, specify that

$\left(\mathrm{a}_{1}\right) 0 x=\sigma_{\gamma_{n}}$, where $x$ is the first element of $\mathbf{x}$ (in the enumeration) which is not involved in any of the diagrams $\mathscr{D}_{1}, \ldots, \mathscr{D}_{n-1}$, and which has not been used to send 0 to any previous $\sigma_{\gamma_{m}}$.

Enumerate $\mathbf{x}_{2}$. Pick a sequence $q_{n} \downarrow-\infty$ of negative rational numbers, and enumerate $\left\{\left(q_{n}\right)_{\gamma} \mid n \in \mathbf{Z}^{+}, \gamma \in \Gamma\right\}$. Proceeding by induction according to this enumeration, specify

$\left(\mathrm{a}_{2}\right)\left(q_{n}\right)_{\gamma} x=\left(1 / q_{2 n}\right)_{\gamma}$ and $0 x=\left(1 / 2 q_{2 n}\right)_{\gamma}$, where $x$ is the first element of $\mathbf{x}_{2}$ which is not involved in any of the diagrams $\mathscr{D}_{m}$ with $\gamma_{m} \leqslant \gamma$, and which has not been used to send any previous $\left(q_{n}\right)_{\gamma}$ to $\left(1 / q_{n}\right)_{\gamma}$.

The specifications thus far involve no loops. Moreover, they preserve the convex congruences of the wreath product, i.e., $(\mu x) \mathscr{C}^{\gamma}(\nu x)$ iff $\mu \mathscr{C}^{\gamma} \nu$ and $(\mu x) \mathscr{C}_{\gamma}(\nu x)$ iff $\mu \mathscr{C}_{\gamma} \nu$. 
The proof is completed by an enumeration of the pairs $(\alpha, z)$ as in Theorem 3, taking care to preserve the properties just mentioned. When specifying an $x^{ \pm 1}$-arrow with tail at $\alpha$, there will necessarily be a largest $\beta_{L}<\alpha$ that is already the tail of an $x^{ \pm 1}$-arrow $\left(\beta_{L}=-\infty\right.$ if there are no such tails), and dually for $\beta_{U}$. Pick that $\gamma$ for which $\beta_{L} \mathscr{C}^{\gamma} \boldsymbol{\alpha}$ or $\alpha \mathscr{C}^{\gamma} \beta_{U}$, but neither $\beta_{L} \mathscr{C}_{\gamma} \alpha$ nor $\alpha \mathscr{C}_{\gamma} \beta_{U}$. Within $\alpha^{\mathscr{C}^{\gamma}}, \beta_{L} \mathscr{C}_{\gamma}$ is the largest $\mathscr{C}_{\gamma}$-class containing a tail less than $\alpha$ of an $x^{ \pm 1}$-arrow (possibly $\beta_{L} \mathscr{C}_{\gamma}=-\infty$, now referring to the lower end of $\alpha \mathscr{C}^{\gamma} / \mathscr{C}_{\gamma}$ ), and dually. As the head of the $x^{ \pm 1}$-arrow being defined, pick any previously unspecified point of $\Omega$ strictly between $\beta_{L}^{\prime} \mathscr{C}_{\gamma}$ and $\beta_{U}^{\prime} \mathscr{C}_{\gamma}\left(\beta_{L}^{\prime}\right.$ being the head of the $x^{ \pm 1}$-arrow with tail $\beta_{L}$, and dually). Such a point must exist because the previous specifications preserve the convex congruences, and because the only limit point in $\Omega$ of previously specified points is 0 .

The case in which $\Gamma$ has a largest element $\bar{\gamma}$ is treated by a similar but easier modification of the proof of [7, Theorem 17], which is left to the reader.

Proof of Theorem 4A. For each $\eta$, the cardinalities are at most those indicated. For $F_{\eta}$ has at most $2^{\omega_{0}}$ prime subgroups, so there are at most $2^{\omega_{0}}$ minimal prime subgroups and thus at most $2^{\omega_{0}}$ roots in $\mathscr{P}_{\eta}$, so $\mathscr{P}_{\eta}$ has at most $2^{\omega_{0}}$ branches.

For any one $\mathscr{P}_{\eta},(2)$ is part of [7, Corollary 16]. Let $P \in \mathscr{P}_{\eta}$. By Lemma 7, $\mathscr{L}(P)$ is $o$-isomorphic to $\mathscr{P}_{\mu}$, where $\mu=\operatorname{group} \operatorname{rank}\left(P \cap G_{\eta}\right) \leqslant \omega_{0}$. Thus every branch $\mathscr{B}$ of $\mathscr{P}$ is $o$-isomorphic to some $\mathscr{P}_{\mu}\left(0 \leqslant \mu \leqslant \omega_{0}\right)$-just apply the previous sentence to the largest element $P$ of $\mathscr{B}$. Since every branch of $\mathscr{R}_{\eta}$ is a branch of $\mathscr{P}_{\eta}$, this applies also to the branches of $\mathscr{R}_{\eta}$. By Lemma $9 \mathrm{~A}$, each such $\mathscr{P}_{\eta}$ occurs $2^{\omega_{0}}$ times as a branch even of $\mathscr{R}_{\eta}$. Therefore both $\mathscr{P}_{\eta}$ and $\mathscr{R}_{\eta}$ have $2^{\omega_{0}}$ branches $o$-isomorphic to $\mathscr{P}_{\mu}$, for each $0 \leqslant \mu \leqslant \omega_{0}$, and no other branches. Since this is true of every finite $\eta>1$, we have established that all these $\mathscr{P}_{\eta}$ 's and $\mathscr{R}_{\eta}$ 's are $o$-isomorphic to each other. Let $\mathscr{P}_{f}$ denote this common root system. We have also established (1), (4), and (3) except for the distinctness of $\mathscr{P}_{f}$ and $\mathscr{P}_{\omega_{0}}$. For that we anticipate part (4) of Theorem 4B and contrast it with (2) of the present theorem.

Because of (2), the chain of covering pairs in any root of $\mathscr{P}_{f}$ (which certainly has cardinality at most that of $F_{\eta}$ ) is as described in (5). Anticipating Theorem 4B again, every nonempty finite or countable chain $\Gamma$ occurs as the chain of covering pairs of some root of $\mathscr{P}_{\omega_{0}}$, and $\mathscr{P}_{\omega_{0}}$ occurs as a branch $\mathscr{B}$ of $\mathscr{P}_{f}$. Letting $P$ be the largest element of $\mathscr{B}$, the pair $\left(P, F_{\eta}\right)$ adds one more element at the top of $\Gamma$ to form the chain of covering pairs of a root of $\mathscr{P}_{f}$. This proves (5) except for singleton chains, which are furnished by the singleton branches.

Proof of Theorems 4B and 4C. We prove 4C, taking for granted those parts that duplicate the proof of $4 \mathrm{~A}$ and pausing intermittently to prove those parts of $4 \mathrm{~B}$ that are not special cases of $4 \mathrm{C}$. We get (3), (2)(a), and (1) as in $4 \mathrm{~A}$, for both $\mathscr{P}_{\eta}$ and $\mathscr{R}_{\eta}$.

In any transitive action of an l-group $F$ on a chain $\Omega$, and for any $\alpha \in \Omega$, a one-to-one order-preserving correspondence between the set of $o$-blocks $\Delta$ containing $\alpha$ and the set of prime subgroups $P$ of $F$ containing the stabilizer $F_{\alpha}$ is given by $\Delta \leftrightarrow F_{\Delta}$, the stabilizer of the $o$-block $\Delta$ [3, Theorem 1.6.2]. Thus [7, Theorem 17] gives (4) for $\mathscr{P}_{\eta}$, and its proof gives (4) for $\mathscr{R}_{\eta}$ for chains $\Gamma$ having no largest element (because in that case each $e \neq w \in F_{\eta}$ moves $o$-blocks in arbitrarily large proper convex congruences). If $\Gamma$ has a largest element $\bar{\gamma}$ and $\Gamma^{\prime}=\Gamma \backslash\{\bar{\gamma}\}$, then we use 
(2)(a) to pick a representing maximal prime subgroup $P$ of $F_{\eta}$ such that $\mathscr{L}(P)$ is $o$-isomorphic to $\mathscr{P}_{\eta}$, and we showed above that the larger root system $\mathscr{P}_{\eta}$ has a root in which the chain of covering pairs is $o$-isomorphic to $\Gamma^{\prime}$. Adjoining the pair $\left(P, F_{\eta}\right)$ at the top of this chain, we have (4) for $\mathscr{R}_{\eta}$.

Applying the proof of [7, Theorem 17] to a chain $\Gamma$ of cofinality $\mu$ produces a representing subgroup $P=F_{0}$ whose branch (in $\mathscr{P}_{\eta}$ or $\mathscr{R}_{\eta}$ ) has cofinality $\mu$. Unless $\Gamma_{1}$ and $\Gamma_{2}$ are cofinally $o$-isomorphic (have $o$-isomorphic upper rays), the branches produced are nonisomorphic because in any one branch, the chains of covering pairs in the various roots are all cofinally $o$-isomorphic. The following lemma proves (2)(b) except for the size of each type.

LEMMA 11. Let $\mu \leqslant \eta$ be regular cardinals. Then there are $2^{\eta}$ chains $\Gamma$ of cofinality $\mu$ and cardinality $\eta$, no two of which are cofinally o-isomorphic.

Proof of Lemma 11. First we treat the case $\mu=\eta$. In an $\alpha$-set $\Gamma^{\prime}$ of cardinality $\omega_{\alpha}=\eta$, choose a cofinal subset $\Gamma^{\prime \prime} o$-isomorphic to the ordinal number $\eta$. Form $\Gamma$ from $\Gamma^{\prime}$ by replacing each element of $\Gamma^{\prime \prime}$ by an ordinal number less than $\eta$, in one-to-one fashion. This gives $2^{\eta} \Gamma$ 's, any one of which is cofinally $o$-isomorphic to only $\eta$ others.

For smaller $\mu$, let $\Gamma^{\prime}$ be an $\alpha$-set of cardinality $\mu$, and $\Gamma^{\prime \prime}$ a subset $o$-isomorphic to $\mu$. Form $\Gamma$ by replacing all elements of $\Gamma^{\prime \prime}$ by the same one of the $2^{\eta}$ chains constructed above. This gives $2^{\eta} \Gamma$ 's.

When the above chain $\Gamma$ has cofinality $\eta$, the proof of [7, Theorem 17] produces branches of cardinality $2^{\eta}$. For if $\Delta$ is any nonsingleton $o$-block containing 0 and if $P=F_{\Delta}$, then $P$ contains a subset of $\mathbf{x}_{2}$ of cardinality $\eta$, so $\operatorname{card}\left(P \cap G_{\eta}\right)=\eta$. This makes $\mathscr{L}(P) o$-isomorphic to $\mathscr{P}_{\eta}$ by Lemma 7 , so that the branch containing $P$ has cardinality $2^{\eta}$. Thus there exist the required number of branches of cofinality $\eta$ and cardinality $2^{\eta}$; perhaps branches of smaller cardinality also occur.

If the cofinality of a branch $\mathscr{B}$ is less than $\eta$, then card $(\mathscr{B})$ must be $2^{\eta}$. For pick a cofinal tower $\left\{P_{i} \mid i \in I\right\}$ in $\mathscr{B}$ of cardinality $\mu$. Then $\mathbf{x}=F_{\eta} \cap \mathbf{x}=\left(\cup_{i} P_{i}\right) \cap \mathbf{x}=$ $\bigcup_{i}\left(P_{i} \cap \mathbf{x}\right)$, so $\operatorname{card}\left(P_{i} \cap \mathbf{x}\right)=\eta$ for some $i$ since there are only $\mu P_{i}$ 's. As above, $\operatorname{card}(\mathscr{B})=2^{\eta}$. This concludes the proof of $(2)(b)$.

We pause to show that in $4 \mathrm{~B}$, all branches $\mathscr{B}$ lacking largest elements have cardinality $2^{\omega_{0}}$. Picking $P_{1}<P_{2}<P_{3} \in \mathscr{B}\left(\mathscr{B}\right.$ has no largest element), $\mathscr{L}\left(P_{3}\right)$ cannot be $o$-isomorphic to $\mathscr{P}_{0}$ or $\mathscr{P}_{1}$ and so by (3) must be $o$-isomorphic to $\mathscr{P}_{f}$ or $\mathscr{P}_{\omega_{0}}$ and thus have cardinality $2^{\omega_{0}}$, making card $(\mathscr{B})=2^{\omega_{0}}$.

Finally, we consider the number of occurrences among the branches (of $\mathscr{P}_{\eta}$ or $\mathscr{R}_{\eta}$ ) of $o$-isomorphism types having no largest element. Let $\mathscr{B}$ be a branch of such a type, and pick a minimal prime subgroup $P \in \mathscr{B}$.

Suppose first that the cofinality of $\mathscr{B}$ and thus of the tower $\mathscr{E}$ of covering pairs of prime subgroups containing $P$ has cofinality $\eta$. By Lemma $8, P \cap G_{\eta}=\{e\}$, so $P \cap \mathbf{x}=\square$. Replacing some elements of $\mathbf{x}$ by their inverses, we may assume that $P x>P$ for all $x \in \mathrm{x}$ according to the order in $F_{\eta} / P$ of these cosets $P x$ (ordering the various $x$ 's in any one coset in any way at all). Because of the cofinality assumption on $\mathscr{E}$, which applies also to the tower of covering pairs of $o$-blocks $\Delta$ containing $P$ in 
the action $\left(F_{\eta}, F_{\eta} / P\right)$, and because $\eta$ is regular, some cofinal subset $\mathbf{z}$ of $\mathbf{x}$ is $o$-isomorphic to the ordinal number $\eta$. Deleting from $\mathbf{z}$ the limit ordinals, we may assume that $\mathbf{y}=\mathbf{x} \backslash \mathbf{z}$ also has cardinality $\eta$. Finally, replacing each $y \in \mathbf{y}$ by its inverse, we may assume that $\mathbf{x}$ is partitioned as $\mathbf{x}=\mathbf{y} \cup \mathbf{z}$, with $P y<P$ for $y \in \mathbf{y}$, $P z>P$ for $z \in \mathbf{z}, \operatorname{card}(\mathbf{y})=\eta$, and $\mathbf{z} o$-isomorphic to $\eta$. Every $\mathbf{z}^{\prime} \subseteq \mathbf{z}$ of cardinality $\eta$ is cofinal in $\mathbf{z}$, i.e., $\left\{P z^{\prime} \mid z^{\prime} \in \mathbf{z}^{\prime}\right\}$ is cofinal in $F_{\eta} / P$, so the convex $l$-subgroup generated by $P \cup \mathbf{z}^{\prime}$ must be all of $F_{\eta}$.

Now suppose that $\varphi_{1}$ and $\varphi_{2}$ are permutations of $\mathbf{x}$ with $\mathbf{u}=\mathbf{z} \varphi_{1} \cap \mathbf{y} \varphi_{2}$ having cardinality $\eta$. Each $\varphi_{i}$ induces an $l$-automorphism $\hat{\varphi}_{i}$ of $F_{\eta}$. We claim that $P \hat{\varphi}_{1}$ and $P \hat{\varphi}_{2}$ together generate $F_{\eta}$, and thus lie in different branches. For $\mathbf{z}^{\prime}=\mathbf{u} \varphi_{1}^{-1}$ is a subset of $\mathbf{z}$ of cardinality $\eta$ (and thus is cofinal), so that $F_{\eta}$ is generated by $P \cup \mathbf{z}^{\prime}$ and thus by $P \hat{\varphi}_{1} \cup \mathbf{z}^{\prime} \varphi_{1}=P \hat{\varphi}_{1} \cup \mathbf{u}$. For $u \in \mathbf{u}$, we have $u \in \mathbf{z} \varphi_{1}$ so that $\left(P \hat{\varphi}_{1}\right) u>$ $P \hat{\varphi}_{1}$ and thus $u \wedge e \in P \hat{\varphi}_{1}$; and $u \in \mathbf{y} \varphi_{2}$ so that $u \vee e \in P \hat{\varphi}_{2}$. Therefore the convex $l$-subgroup generated by $P \hat{\varphi}_{1}$ and $P \hat{\varphi}_{2}$ contains $P \hat{\varphi}_{1} \cup \mathbf{u}$, and thus is $F_{\eta}$, proving the claim.

To get $2^{\eta} \varphi_{i}$ 's such that $\operatorname{card}\left(\mathbf{z} \varphi_{i_{1}} \cap \mathbf{y} \varphi_{i_{2}}\right)=\eta$ when $i_{1} \neq i_{2}$, and thus get $2^{\eta}$ branches $o$-isomorphic to $\mathscr{B}$, we use

Lemma 12 (The Pie Lemma). Let $\eta=\omega_{0}$, or (with G.C.H.) let $\eta$ be regular. Let $\Pi$ be a set of cardinality $\eta$. Then $\Pi$ can be partitioned in $2^{\eta}$ ways as $\Pi=A_{i} \cup B_{i}$, and so that when $i \neq j$, card $\left(A_{i} \cap A_{j}\right)=\operatorname{card}\left(A_{i} \cap B_{j}\right)=\operatorname{card}\left(B_{i} \cap A_{j}\right)=\operatorname{card}\left(B_{i} \cap B_{j}\right)$ $=\eta$.

Proof of The Pie Lemma. First we give the proof for $\eta=\omega_{0}$. In a circular pie with center at the origin, let $\Pi$ be the rational pie, i.e., the set of points whose polar coordinates $r(\neq 0)$ and $\theta(0 \leqslant \theta<2 \pi)$ are both rational. For each of the $2^{\omega_{0}}$ numbers $\alpha(0<\alpha<\pi)$ such that $\theta$ and $\theta+\pi$ are both irrational, partition $\Pi$ by cutting the pie along the diameter $\theta=\alpha$.

For higher cardinalities, we rephrase this argument. Let $\Pi$ be an $\alpha$-set of cardinality $\eta$, and let $\Pi$ be the disjoint union $\Lambda_{1} \cup \Lambda_{2}$ of two copies of $\Lambda$. For $\bar{\lambda} \in \bar{\Lambda} / \Lambda$, let

$$
A_{\bar{\lambda}}=\left\{\lambda \in \Lambda_{1} \mid \lambda>\bar{\lambda}\right\} \cup\left\{\lambda \in \Lambda_{2} \mid \lambda<\bar{\lambda}\right\}
$$

and $B_{\bar{\lambda}}$ its complement in $\Pi$. There are $2^{\eta}$ such $\bar{\lambda}$ 's [3, p. 188].

Now suppose that the tower $\mathscr{E}$ above $P$ has cofinality $\mu<\eta$. Then as in the argument about card $(\mathscr{B})$, some $Q \in \mathscr{B}$ contains $\eta$ elements of $\mathbf{x}$. Let $\mathbf{y}=\mathbf{x} \cap Q$ and $\mathbf{z}=\mathbf{x} \backslash \mathbf{y} \cdot \operatorname{card}(\mathbf{z}) \leqslant \operatorname{card}(\mathbf{y})=\eta$. Partition $\mathbf{x}$ into $\eta$ cells $A_{i}$, each having the same cardinality as $\mathbf{z}$. For each $A_{i}$, pick a permutation $\varphi_{i}$ of $\mathbf{x}$ such that $\mathbf{z} \varphi_{i}=A_{i}$ (and thus $\left.\mathbf{y} \varphi_{i}=\mathbf{x} \backslash A_{i}\right)$. For $i_{1} \neq i_{2}$, the convex $l$-subgroup generated by $Q \hat{\varphi}_{i_{1}}$ and $Q \hat{\varphi}_{i_{2}}$ contains $\mathbf{y} \varphi_{1} \cup \mathbf{y} \varphi_{2} \supseteq \mathbf{y} \varphi_{1} \cup \mathbf{z} \varphi_{1}=\mathbf{x} \varphi_{1}$ and thus is all of $F_{\eta}$. Therefore $Q \hat{\varphi}_{i_{1}}$ and $Q \hat{\varphi}_{i_{2}}$ lie in different branches, so this must also be true of $P \hat{\varphi}_{i_{1}}$ and $P \hat{\varphi}_{i_{2}}$. This concludes the proof of Theorems $4 \mathrm{~B}$ and $4 \mathrm{C}$.

4. Right orderings of the free group $G_{\eta}$. Now we apply the foregoing results to $G_{\eta}$, making use of the fact that in the right regular representation of $G_{\eta}$ on any right ordering $\left(G_{\eta}, \leqslant\right)$, the $o$-blocks containing $e$ are precisely the convex subgroups. 
Corollary 13. Let $1<\eta \leqslant \omega_{0}$, or (with G.C.H.) let $\eta$ be regular. Then $G_{\eta}$ has a right ordering in which there are no proper convex subgroups.

Proof. Use the right ordering produced in Theorem 3 (see the discussion prior to that theorem).

We remark that for $\eta \leqslant \omega_{0}$, every $\operatorname{such}\left(G_{\eta}, \leqslant\right)$ must as a chain be $o$-isomorphic to $\mathbf{Q}$ (being countable, and being dense in itself since otherwise there would be a convex cyclic subgroup). For regular $\eta$, the chain $\left(G_{\eta}, \leqslant\right)$ can be chosen to be an $\alpha$-set.

In both cases, $\left(G_{\eta}, \leqslant\right)$ can be chosen to be extremely "stretchable" in the sense that for all $g_{1}<g_{2}<g_{3}<g_{4}$, there exists $g \in G_{\eta}$ such that $g_{2} g<g_{1}$ and $g_{3} g>g_{4}$. This is automatic when $\left(G_{\eta}, \leqslant\right)$ is a representing right ordering lacking proper convex subgroups. For when the natural action of $F_{\eta}$ on $\left(G_{\eta}, \leqslant\right)$ is faithful, it must be $o$-2-transitive [7, Proposition 13]. So given $g_{1}<g_{2}<g_{3}<g_{4}$, there exists $w \in F_{\eta}$ such that $g_{2} w<g_{1}$ and $g_{3} w>g_{4}$. Then $g_{4}<g_{3} w=g_{3}\left(\bigvee_{i} \wedge_{j} w_{i j}\right)=\max _{i} \min _{j} g_{3} w_{i j}$ $=\min _{j} g_{3} w_{i^{\prime} j}$ for some $i^{\prime}$. Now $g_{1}>g_{2} w \geqslant \min _{j} g_{2} w_{i^{\prime} j}$, so $g_{1}>\min g_{2} w_{i^{\prime} j^{\prime}}$ for some $w_{i^{\prime} j^{\prime}}$. Take $g=w_{i^{\prime} j^{\prime}}$.

For the next pair of corollaries, similar remarks can be made about the covering pairs of convex subgroups. For simplicity, we assume small rank $\eta$ in these results.

Corollary 14. Let $1<\eta \leqslant \omega_{0}$. Let $n \in \mathbf{Z}^{+}$. Then $G_{\eta}$ has right ordering in which there are precisely $n$ proper convex subgroups $K_{1} \subset \cdots \subset K_{n}$. Moreover, $\mu_{i}=$ $\operatorname{rank}\left(K_{i}\right)$ can be prescribed $\left(1<\mu_{i} \leqslant \omega_{0}\right.$, except that $\mu_{1}=1$ is permitted $)$, and the conjugates of each $K_{i}$ in $K_{i+1}$ can be made to have trivial intersection (where $K_{n+1}=G_{\eta}$ ).

Proof. By induction, there exists a right ordering $\left(G_{\eta}, \leqslant^{\prime}\right)$ having precisely $n-1$ convex subgroups $K_{2} \subset \cdots \subset K_{n}$, with $\operatorname{rank}\left(K_{i}\right)=\mu_{i}$, and with the intersection of the conjugates of $K_{i}$ in $K_{i+1}$ trivial. (For $n=1$, use Corollary 13.) We want to produce a right ordering $\left(K_{2}, \preccurlyeq\right)$ having precisely one convex subgroup $K_{1}$ (of rank $\mu_{1}$ ), and such that the intersection of the conjugates of $K_{1}$ in $K_{2}$ is trivial. Then an application of Theorem 2 to the natural action of $F_{\eta}$ on the induced right ordering $\left(G_{\eta} / K_{2}, \leqslant^{\prime}\right)$, with $\left(K_{2}, \preccurlyeq\right)$ as the ordering of the stabilizer $K_{2}$, will produce the desired right ordering $\left(G_{\eta}, \leqslant\right)$. (The convex subgroups above $K_{2}$ will be the same for $\left(G_{\eta}, \leqslant\right)$ as for $\left(G_{\eta}, \leqslant^{\prime}\right)$.)

To produce $\left(K_{2}, \preccurlyeq\right)$, or equivalently $\left(G_{\mu_{2}}, \preccurlyeq\right)$, we apply Lemma 9A to $F=F_{\mu_{2}}$ (since $\mu_{2}>1$ ) to obtain a representing maximal prime subgroup $P$ such that $\operatorname{rank}\left(P \cap G_{\mu_{2}}\right)=\mu_{1}$ (cf. Lemma 6). By Corollary 13, we choose a right ordering ( $P \cap G_{\mu_{2}}, \preccurlyeq^{\prime}$ ) having no proper convex subgroups. We apply Theorem 2 to the representation $(F, F / P)$ and to $\left(P \cap G_{\mu_{2}}, \preccurlyeq^{\prime}\right)$. Since $(F, F / P)$ has no proper $o$-blocks, the resulting right ordering $\left(G_{\mu_{2}}, \preccurlyeq\right)$ has the desired properties.

COROllary 15. Let $1<\eta \leqslant \omega_{0}$. Let $\Gamma$ be any nonempty finite or countable chain. Then $G_{\eta}$ has a right ordering in which the chain of covering pairs $\left(K_{\gamma}, K^{\gamma}\right)$ of convex subgroups is o-isomorphic to $\Gamma$.

Proof. Use part (4) of Theorem 4B. 


\section{5. l-automorphisms of $F_{\eta}$.}

LEMMA 16. A one-to-one correspondence between the set of minimal prime subgroups of $F_{\eta}$ and the set of right orderings $\left(G_{\eta}, \leqslant\right)$ is given by

$$
P \rightarrow\left(g_{1} \leqslant g_{2} \Leftrightarrow P g_{1} \leqslant P g_{2}\right) \text {. }
$$

The inverse correspondence is $\left(G_{\eta}, \leqslant\right) \rightarrow F_{e}$, where $F_{e}$ is the stabilizer of $e$ in the natural action of $F=F_{\eta}$ on $\left(G_{\eta}, \leqslant\right)$.

Proof. Let $P$ be a minimal prime subgroup of $F_{\eta}$. Then $P \cap G_{\eta}=\{e\}$ by Lemma 8, so " $g_{1} \leqslant g_{2}$ iff $P g_{1} \leqslant P g_{2}$ " is a right ordering $\left(G_{\eta}, \leqslant\right)$. Since the natural action of $F_{\eta}$ on $\left(G_{\eta}, \leqslant\right)$ coincides with its action on $F_{\eta} / P$ by Lemma $5, F_{e}=P_{\eta}$.

Going the other way, consider a right ordering $\left(G_{\eta}, \leqslant\right)$. In the natural action of $F_{\eta}$ on $\left(G_{\eta}, \leqslant\right), F_{e} \cap G_{\eta}=\{e\}$. By Lemma $8, P=F_{e}$ is a minimal prime subgroup of $F$. Clearly the right ordering associated with $P$ is the given one $\left(G_{\eta}, \leqslant\right)$.

THEOREM 17. Let $P$ be a minimal prime subgroup of $F_{\eta}$. Then the number of l-automorphic images of $P$ is

(1) $\omega_{0}$ (if $\left.1<\eta<\omega_{0}\right)$.

(2) $2^{\eta}$ (if $\eta$ is infinite).

Proof. In each case, the number of $l$-automorphisms and thus of $l$-automorphic images of $P$ is at most as indicated. (Consider the effect of an $l$-automorphism on $\mathbf{x}$.)

By Lemma 16, $P$ is the stabilizer $F_{e}$ in the natural action of $F=F_{\eta}$ on some right ordering $\left(G_{\eta}, \leqslant\right)$. Let $G_{\eta}^{+}$denote the positive cone. For an $l$-automorphism $\varphi$ of $F_{\eta}$, $P \varphi \leftrightarrow\left(G_{\eta}, \leqslant_{\varphi}\right)$, the right ordering having $G_{\eta}^{+} \varphi$ as its positive cone. We shall produce the desired number of $l$-automorphisms $\varphi$ yielding distinct images $G_{\eta}^{+} \varphi$.

For infinite $\eta$, the proof is reminiscent of the last part of the proof of Theorems 4B and 4C. By the minimality of $P, P \cap G_{\eta}=\{e\}$, so $P \cap \mathbf{x}=\square$. We may assume that $P<P x$ for all $x \in \mathbf{x}$. For each $\mathbf{y} \subseteq \mathbf{x}$, let $\varphi_{\mathbf{y}}(x)=x^{-1}$ if $x \in \mathbf{y}$ and $\varphi_{\mathbf{y}}(x)=x$ if $x \in \mathbf{x} \backslash \mathbf{y}$. Extend $\varphi_{\mathbf{y}}$ to an $l$-automorphism of $F_{\eta}$. These $2^{\eta} l$-automorphisms do the trick.

For finite $\eta$, we have $\mathbf{x}=\left\{x_{1}, \ldots, x_{\eta}\right\}$ and we select $\omega_{0}$ sets $z=\left\{z_{1}, \ldots, z_{\eta}\right\}$ which are free generating sets of the group $G_{\eta}$ and thus also of the $l$-group $F_{\eta}$. The function $x_{i} \rightarrow z_{i}$ can be extended to an $l$-automorphism $\varphi_{z}$ of $F_{\eta}$. We select the z's carefully, so that the images $G_{\eta}^{+} \varphi_{z}^{-1}$ are distinct.

With no loss of generality, $\mathbf{x}=\{x, y, \ldots\}$, with $e<x<y$. Changing one element of $\mathbf{x}$ by multiplying it by a different element of $\mathbf{x}$ yields another free generating set $\mathbf{x}^{\prime}$ for $G_{\eta}$ [5]. Accordingly, we may take $\mathbf{z}_{n}=\left\{z_{n 1}, z_{n 2}, \ldots\right\}$ to be $\left\{y, x y^{n}, \ldots\right\}$. Since $e<x<y$, we have $y^{n}<x y^{n}<y^{n+1}$, i.e., $z_{n 1}^{n}<z_{n 2}<z_{n 1}^{n+1}$. Thus $z_{n 2} z_{n 1}^{-n} \in G_{\eta}^{+}$but $z_{n 2^{2}} z_{n 1}^{-(n+1)} \notin G_{\eta}^{+}$. Therefore $\left(y x^{-n}\right) \varphi_{z_{n}} \in G_{\eta}^{+}$but $\left(y x^{-(n+1)}\right) \varphi_{z_{n}} \notin G_{\eta}^{+}$, making the images $G_{\eta}^{+} \varphi_{z_{n}}^{-1}$ distinct.

Just "how unbounded" the supports of various elements are in a pathologically $o$-2-transitive representation $\left(F_{\eta}, \Omega\right)$ can vary. Of course, there cannot be both a 
nonidentity element with support bounded above and another with support bounded below. However

THEOREM 18. $F_{\eta}(\eta>1)$ has pathologically o-2-transitive representations of each of the following kinds:

(1) No nonidentity element has support bounded either way. (For $3 \leqslant \eta \leqslant \omega_{0}$, or (with G.C.H.) $\eta$ regular, it can be further arranged that no nonidentity group word fix any point.)

(2) All elements have support bounded below (resp., above). (Valid for infinite $\eta$, but false for finite $\eta$.)

(3) At least one free generator $x \in \mathbf{x}$ has support bounded below (resp., above), and at least one does not.

Proof. We modify the proof of [7, Theorem 1], for now with $\eta$ finite.

For (1), we lay out a diagram in each interval $[2 n, 2 n+1]$, but for all (not just positive) $n$, arranging that for each $e \neq w \in F_{\eta}$ the set of diagrams for $w$ be coterminal in $\mathbf{Q}$. We build bridges just as before, except for the bridge in $[1,2]$. There, we arrange that the free generator $x_{t_{0}}$ moving 1 and the free generator $x_{r_{1}}$ moving 2 be distinct. (No $x$ has been specified as fixing 2, for then the $x$-arrow from 2 to 2 would have formed a loop, whereas diagrams are loop-free. Thus $x^{ \pm 1}$ may be specified to move 2 down without violating consistency.) We specify as before that $1 x_{t_{0}}^{ \pm 1}=4 / 3,(5 / 3) x_{r_{1}}^{ \pm 1}=2$, and $(4 / 3) x_{t_{0}}^{ \pm 1}=5 / 3$. But we also specify that $(4 / 3+1 /(n+1)) x_{r_{1}}^{ \pm 1}=4 / 3+1 / n$ for $n \geqslant 3$, and that all free generators except $x_{t_{0}}$ fix $4 / 3$.

Again the (transitive) action of $F_{\eta}$ on $\Omega=0 F_{\eta}$ is faithful. Moreover, this representation is $o$-primitive. For let $\Delta$ be a nonsingleton $o$-block containing $4 / 3$. On account of $x_{r_{1}}, \Delta$ must contain $5 / 3$, and then $\Delta x=\Delta$ for all free generators $x$, forcing $\Delta=\Omega$. Now [7, Proposition 13] guarantees that this representation is pathologically $o-2$ transitive. (1) obtains by construction.

For $\eta \geqslant 3$, we can sharpen the preceding argument by constructing the bridge in $[1,2]$ according to the proof of Theorem 3. Pick an irrational number $r, 4 / 3<r<$ $5 / 3$, and a third free generator $x\left(\neq x_{t_{0}}, x_{r_{1}}\right)$. Lay out in the interval $(4 / 3, r)$ the specifications (through (e)) made in the proof of Theorem 3 for $(0, \infty)$, with $x_{t_{0}}^{\mp 1}$ playing the role played before by $y$, and the present $x$ playing the same role as the previous $x$. Pick a rational $\beta, 4 / 3<\beta<r$, and specify that $(4 / 3) x_{r_{1}}^{ \pm 1}=\beta$ and $\beta x_{r_{1}}^{ \pm 1}=2$. Specify also that the free generators other than these three move $4 / 3$ to distinct "new" points between $4 / 3$ and 2. Enumerate as in the proof of Theorem 3 .

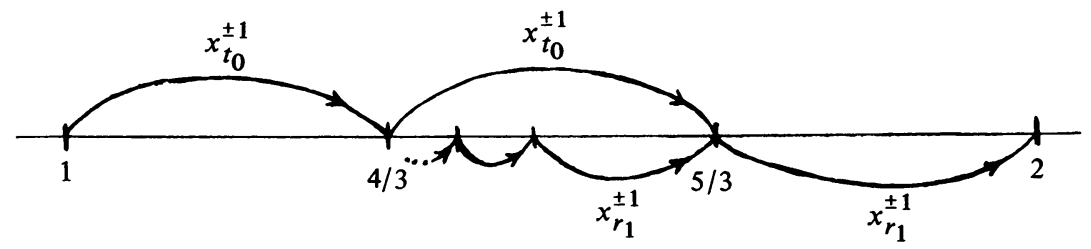

FIGURE 7 
As above, $\left(F_{\eta}, \Omega\right)$ is pathologically $o$-2-transitive. Whether the restriction that $\eta \neq 2$ is necessary is an open question.

(2) follows from [7, Lemma 14]. For (3), we simply arrange in the unmodified proof of [7, Theorem 1] that $\operatorname{support}\left(x_{r_{0}}\right)$ be bounded below, whereas $\operatorname{support}\left(x^{\prime}\right)$ not be.

For infinite $\eta$, the appropriate modifications in the proof of [7, Theorem 1] should now be obvious. (For (2), if all free generators have support bounded below, so do all elements of $F_{\eta}$.)

Remark. Since $F_{\omega_{0}}$ can be represented in all three ways just described in (1) and (2), pathologically $o$-2-transitive $l$-permutation groups of these three types cannot be discriminated from one another in l-group language.

The various maximal prime subgroups of $F_{\eta}$ which we have constructed do not all "appear" in any one transitive representation of $F_{\eta}$ :

COROLlARY 19. There is no transitive representation $\left(F_{\eta}, \Omega\right)$ for which the stabilizers $\left(F_{\eta}\right)_{\bar{\omega}}, \bar{\omega} \in \bar{\Omega}$, include all representing maximal prime subgroups of $F_{\eta}$.

Proof. Let $F=F_{\eta}$. First, no pathologically $o$-2-transitive representation $(F, \Omega)$ of type (3) can do this. For suppose one does. The stabilizers $F_{\bar{\omega}}$ must be precisely the representing maximal prime subgroups of $F$. Since stabilizers of distinct cuts $\bar{\omega} \in \bar{\Omega}$ are distinct, every $l$-automorphism $\varphi$ of $F$ induces a permutation $\bar{\varphi}$ of $\bar{\Omega}(\bar{\omega} \bar{\varphi}=\bar{\alpha}$, where $F_{\bar{\omega}} \varphi=F_{\bar{\alpha}}$ ). $\bar{\varphi}$ preserves order because $\bar{\omega}_{1}<\bar{\omega}_{2}$ iff there exists $f \in F$ (namely an appropriate $f$ whose support is bounded below-cf. (3)) such that $f \in F_{\bar{\omega}_{2}}$ and $f^{h} \in F_{\bar{\omega}_{1}}$ for every $e<h \in F$. Hence for $h \in F, h \varphi$ has support bounded below (i.e., $h \varphi \in F_{\bar{\omega}}$ for all sufficiently small $\bar{\omega}$ ) iff the same is true of $h$. But any free generator of $F$ can be sent to any other by some $l$-automorphism $\varphi$. For free generators of the two kinds in (3), this gives a contradiction.

Now suppose some other transitive representation $(F, \Omega)$ has the property in question. Let $P$ be the stabilizer of a point in some representation of type (3). The property guarantees first that $P=F_{\bar{\omega}}$ for some $\bar{\omega} \in \bar{\Omega}$, and then that every representing maximal prime subgroup $Q=F_{\bar{\alpha}}$ for some $\bar{\alpha} \in \bar{\Omega}$. The latter condition makes $Q \subseteq F_{\Delta}$, where $\Delta$ is the largest segment of $\bar{\Omega}$ containing $\bar{\alpha}$ and not meeting the orbit $\bar{\omega} F(\Delta=\{\bar{\alpha}\}$ if $\bar{\alpha} \in \bar{\omega} F)$. But $F_{\Delta}$ is the stabilizer of a cut in $\bar{\omega} F$, and the representation on $\bar{\omega} F$ is $o$-2-transitive, so $F_{\Delta}$ is a representing subgroup of $F$. The maximality of $Q$ makes $Q=F_{\Delta}$. This means that every representing maximal prime subgroup $Q$ of $F$ is the stabilizer of a cut in $(F, F / P)$, contradicting the first part of the proof.

For various classes of transitive $l$-permutation groups $(H, \Omega)$, the $l$-automorphisms of $H$ have been related to the stabilizers $H_{\bar{\omega}}(\bar{\omega} \in \bar{\Omega})$ roughly as in the proof of Corollary 19, with the conclusion that every $l$-automorphism of $H$ is induced by conjugation by some element of $A(\bar{\Omega})$. Specifically this has been shown for all $o$-primitive $l$-permutation groups except those which are pathologically $o$-2-transitive [3, Corollary 7E]. Attempts to counterexample conjectures of this kind founder on the lack of other ways of producing $l$-automorphisms. But since in $F_{\eta}$ any free 
generator can be sent by an $l$-automorphism to any other, case (3) of Theorem 18 gives

CountereXample 20. $F_{\eta}(\eta>1)$ has a pathologically $o$-2-transitive representation $\left(F_{\eta}, \Omega\right)$ for which there exist $l$-automorphisms of $F_{\eta}$ not induced by conjugation by elements of $A(\bar{\Omega})$.

\section{REFERENCES}

1. A. Kumar Arora and S. H. McCleary, Centralizers in free lattice-ordered groups, Houston J. Math. (to appear).

2. P. Conrad, Free lattice-ordered groups, J. Algebra 16 (1970), 191-203.

3. A. M. W. Glass, Ordered permutation groups, London Math. Soc. Lecture Note Ser. 55, Cambridge Univ. Press, London and New York, 1981.

4. V. M. Kopytov, Free lattice-ordered groups, Algebra and Logic 18 (1979), 259-270. (English translation)

5. R. C. Lyndon and P. E. Schupp, Combinatorial group theory, Springer-Verlag, Berlin and New York, 1976.

6. S. H. McCleary, O-2-transitive ordered permutation groups, Pacific J. Math 49 (1973), 425-429.

7. Free lattice-ordered groups represented as o-2-transitive l-permutation groups, Trans. Amer. Math. Soc. 290 (1985), 69-79.

Department of Mathematics, University of Georgia, Athens, Georgia 30602 